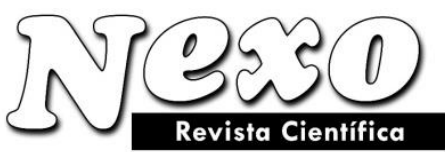

Vol. 34, No. 02, pp. 636-660/Junio 2021

\title{
Diseño térmico de un intercambiador de calor de doble tubo aleteado para el enfriamiento de metanol
}

\section{Thermal design of a finned double pipe heat exchanger for methanol cooling}

\author{
Amaury Pérez Sánchez ${ }^{1, *}$, Greisy Ivety Valero Almanza² ${ }^{2}$ Elizabeth Ranero González ${ }^{1}$, Eddy \\ Javier Pérez Sánchez ${ }^{3}$, \\ ${ }^{1}$ Universidad de Camagüey, Facultad de Ciencias Aplicadas, Camagüey, Cuba. \\ ${ }^{2}$ Centro de Ingeniería Ambiental de Camagüey, Departamento de Gestión Ambiental, Camagüey, Cuba. \\ ${ }^{3}$ Empresa de Servicios Automotores S.A., Dirección Comercial, Ciego de Ávila, Cuba. \\ *amauryps@nauta.cu
}

(recibido/received: 14-octubre-2020; aceptado/accepted: 11-diciembre-2020)

\section{RESUMEN}

En el presente trabajo se efectuó el diseño térmico de un intercambiador de calor de doble tubo con tubos aleteados para efectuar el enfriamiento de metanol. Se realizaron además dos estudios de sensibilidad para determinar la influencia que presenta un incremento de tanto el caudal de alimentación $\left(\mathrm{m}_{\mathrm{c}}\right)$ como la temperatura de entrada del metanol $\left(T_{1}\right)$ sobre cuatro parámetros de diseño del intercambiador. El intercambiador de calor diseñado tendrá una eficiencia de la aleta de 0,236, un factor de limpieza de 0,60, un área de transferencia de calor bajo condiciones de ensuciamiento de $20,53 \mathrm{~m}^{2}$ y un número total de horquillas igual a tres para cumplir con la demanda térmica del sistema. Tanto la caída de presión calculada del agua de enfriamiento $(5880,39 \mathrm{~Pa})$ como la del metanol $(70711,91 \mathrm{~Pa})$ se encuentran por debajo de los límites máximos fijados por el proceso. Se necesita una potencia de bombeo de 17,52 W y $160,62 \mathrm{~W}$ para impulsar el agua de enfriamiento y el metanol respectivamente. Se recomienda que $\mathrm{m}_{\mathrm{c}}$ no supere los $5800 \mathrm{~kg} / \mathrm{h}$, mientras que $\mathrm{T}_{1}$ puede incrementarse hasta $80^{\circ} \mathrm{C}$ sin que esto afecte negativamente la caída de presión del agua de enfriamiento, aunque esto incrementa la potencia de bombeo del agua de enfriamiento.

Palabras claves: Caída de presión; Diseño térmico; Horquillas; Intercambiador de calor, Potencia de bombeo.

\begin{abstract}
In the present work, the thermal design of a double tube heat exchanger with finned tubes for methanol cooling was performed. Two sensitivity studies were also carried out to determine the influence of an increase in both the feed rate $\left(\mathrm{m}_{\mathrm{c}}\right)$ and the methanol inlet temperature $\left(\mathrm{T}_{1}\right)$ on four exchanger design parameters. The designed heat exchanger will have a fin efficiency of 0.236 , a cleaning factor of 0.60 , a heat transfer area under fouling conditions of $20.53 \mathrm{~m}^{2}$, and a total number of hairpins equal to three to meet the thermal duty of the system. Both the calculated pressure drop of the cooling water $(5,880.39 \mathrm{~Pa})$ and the methanol $(70,711.91 \mathrm{~Pa})$ are below the maximum limits established by the process. A pumping power of $17.52 \mathrm{~W}$ and $160.62 \mathrm{~W}$ is required to pump the cooling water and methanol respectively. It is recommended that $m_{c}$ does not exceed $5,800 \mathrm{~kg} / \mathrm{h}$, while $\mathrm{T}_{1}$ can be increased up to $80{ }^{\circ} \mathrm{C}$ without
\end{abstract}


negatively affecting the pressure drop of the cooling water, although this increases the pumping power of the cooling water.

Keywords: Pressure drop; Thermal Design; Hairpins; Heat Exchanger; Pumping Power.

\section{NOMENCLATURA}

\begin{tabular}{|c|c|c|}
\hline$a_{t}$ & Área de flujo del tubo interior & $\mathrm{m}^{2}$ \\
\hline $\mathrm{A}_{\mathrm{al}}$ & Área de transferencia de calor aleteada & $\mathrm{m}^{2}$ \\
\hline $\mathrm{A}_{\mathrm{h}}$ & Área total de una horquilla & $\mathrm{m}^{2}$ \\
\hline$A_{i}$ & Área interna & $\mathrm{m}^{2}$ \\
\hline$A_{L}$ & Área total de transferencia de calor sin considerar ensuciamiento & $\mathrm{m}^{2}$ \\
\hline$A_{n}$ & Área de transferencia de calor no aleteada & $\mathrm{m}^{2}$ \\
\hline$A_{n t}$ & Área neta transversal del ánulo con tubos aleteados longitudinales & $\mathrm{m}^{2}$ \\
\hline$A_{S}$ & Área total de transferencia de calor bajo condiciones de ensuciamiento & $\mathrm{m}^{2}$ \\
\hline $\mathrm{Cp}$ & Calor especifico & $\mathrm{J} / \mathrm{kg} \cdot \mathrm{K}$ \\
\hline$d_{e}$ & Diámetro exterior del tubo interior & $\mathrm{m}$ \\
\hline $\mathrm{d}_{\mathrm{i}}$ & Diámetro interior del tubo interior & $\mathrm{m}$ \\
\hline$d_{n}$ & Diámetro nominal del tubo interior & pulg \\
\hline $\mathrm{D}_{\mathrm{eq}}$ & Diámetro equivalente para la transferencia de calor & $\mathrm{m}$ \\
\hline $\mathrm{D}_{\mathrm{h}}$ & Diámetro hidráulico & $\mathrm{m}$ \\
\hline $\mathrm{D}_{\mathrm{i}}$ & Diámetro interior del ánulo & $\mathrm{m}$ \\
\hline$D_{n}$ & Diámetro nominal del ánulo & pulg \\
\hline $\mathrm{e}_{\mathrm{al}}$ & Espesor de la aleta & $\mathrm{m}$ \\
\hline $\mathrm{f}$ & Factor de fricción de Fanning & - \\
\hline $\mathrm{f}^{\prime}$ & Factor de fricción de Fanning corregido & - \\
\hline $\mathrm{F}_{\mathrm{L}}$ & Factor de limpieza & - \\
\hline $\mathrm{h}$ & Coeficiente pelicular de transferencia de calor & $\mathrm{W} / \mathrm{m}^{2} . \mathrm{K}$ \\
\hline $\mathrm{H}_{\mathrm{al}}$ & Altura de la aleta & $\mathrm{m}$ \\
\hline $\mathrm{k}$ & Conductividad térmica & $\mathrm{W} / \mathrm{m} . \mathrm{K}$ \\
\hline $\mathrm{k}_{\mathrm{t}}$ & Conductividad térmica material del tubo interior & $\mathrm{W} / \mathrm{m} \cdot \mathrm{K}$ \\
\hline $\mathrm{L}_{\mathrm{t}}$ & Longitud del tubo & $\mathrm{m}$ \\
\hline $\mathrm{m}$ & Parámetro & - \\
\hline $\mathrm{m}$ & Caudal másico & $\mathrm{kg} / \mathrm{s}$ \\
\hline MLDT & Media Logarítmica de la Diferencia de Temperatura & ${ }^{\circ} \mathrm{C}$ \\
\hline $\mathrm{N}_{\mathrm{al}}$ & Número de aletas por tubo & - \\
\hline $\mathrm{Nh}$ & Número de horquillas & - \\
\hline $\mathrm{N}_{\mathrm{t}}$ & Numero de tubos & - \\
\hline $\mathrm{Nu}$ & Número de Nusselt & - \\
\hline $\mathrm{P}_{\mathrm{b}}$ & Potencia de bombeo & $\mathrm{W}$ \\
\hline $\mathrm{P}_{\mathrm{h}}$ & $\begin{array}{c}\text { Perímetro húmedo total del ánulo con tubos interiores aleteados de forma } \\
\text { longitudinal }\end{array}$ & $\mathrm{m}$ \\
\hline $\mathrm{P}_{\mathrm{hc}}$ & Perímetro húmedo para la transferencia de calor & $\mathrm{m}$ \\
\hline $\operatorname{Pr}$ & Número de Prandtl & - \\
\hline$\Delta \mathrm{p}$ & Caída de presión & $\mathrm{Pa}$ \\
\hline$\Delta \mathrm{P}_{(\mathrm{p})}$ & Caída de presión máxima permisible & $\mathrm{Pa}$ \\
\hline Q & Carga de calor o calor intercambiado & $\mathrm{W}$ \\
\hline $\mathrm{R}$ & Factor de ensuciamiento & $\mathrm{m}^{2} . \mathrm{K} / \mathrm{W}$ \\
\hline $\mathrm{Re}$ & Número de Reynolds & - \\
\hline $\mathrm{t}$ & Temperatura del fluido frío & ${ }^{\circ} \mathrm{C}$ \\
\hline
\end{tabular}




$\begin{array}{ccc}\mathrm{T} & \text { Temperatura del fluido caliente } & { }^{\circ} \mathrm{C} \\ \mathrm{T}_{\mathrm{p}} & \text { Temperatura de la pared del tubo } & { }^{\circ} \mathrm{C} \\ \bar{t} & \text { Temperatura media del fluido frío } & { }^{\circ} \mathrm{C} \\ \bar{T} & \text { Temperatura media del fluido caliente } & { }^{\circ} \mathrm{C} \\ \mathrm{U}_{\mathrm{L}} & \text { Coeficiente global de transferencia de calor limpio } & \mathrm{W} / \mathrm{m}^{2} . \mathrm{K} \\ \mathrm{U}_{\mathrm{S}} & \text { Coeficiente global de transferencia de calor sucio } & \mathrm{W} / \mathrm{m}^{2} . \mathrm{K} \\ \mathrm{v} & \text { Velocidad } & \mathrm{m} / \mathrm{s}\end{array}$

\section{Símbolos griegos}

$\begin{array}{cc}\rho & \text { Densidad } \\ \mu & \text { Viscosidad } \\ \eta_{\mathrm{al}} & \text { Eficiencia de la aleta } \\ \eta_{\mathrm{b}} & \text { Eficiencia isoentrópica de la bomba } \\ \eta_{\mathrm{s}} & \text { Eficiencia global superficial } \\ & \text { Subíndices } \\ 1 & \text { Entrada } \\ 2 & \text { Salida } \\ \mathrm{a} & \text { Del fluido que circula por el ánulo } \\ \mathrm{c} & \text { Del fluido caliente } \\ \mathrm{f} & \text { Del fluido frío } \\ \text { lam } & \text { Bajo régimen laminar } \\ \mathrm{p} & \text { A la temperatura de la pared del tubo interior } \\ \mathrm{t} & \text { Del fluido que circula por el tubo interior } \\ \text { turb } & \text { Bajo régimen turbulento }\end{array}$

\section{INTRODUCCIÓN}

La mejora de la transferencia de calor en un intercambiador de calor está adquiriendo importancia industrial debido a que suministra la oportunidad de reducir el área de transferencia de calor para el intercambiador de calor. El incremento del rendimiento del intercambiador de calor puede ayudar a obtener ahorros de material, energía y costo relacionados con el proceso de intercambio de calor (Kumar et al., 2015).

Un intercambiador de calor es un dispositivo de transferencia de calor que posibilita el intercambio de calor entre dos o más fluidos de proceso. Presenta un extenso uso en aplicaciones industriales y domésticas. Se han desarrollado varios tipos de intercambiadores de calor para su empleo en plantas termoenergéticas, plantas químicas, sistemas de acondicionamiento de aire, unidades de refrigeración, entre otras (Zohuri, 2017).

Uno de los intercambiadores de calor más empleados en la industria química actual es el de doble tubo, también denominados de horquilla. Un intercambiador de calor de doble tubo típico consiste de una tubería ubicada de forma concéntrica por dentro de otra tubería de mayor diámetro con aditamentos apropiados para dirigir el flujo de una sección hacia otra (Fig. 1). Un fluido fluye por la tubería interior (lado del tubo), y el otro fluye a través del espacio anular (ánulo). El empleo principal que presentan los intercambiadores de calor de doble tubo radica en el calentamiento o enfriamiento de fluidos de proceso donde se requieran área de transferencia de calor pequeñas (hasta $50 \mathrm{~m}^{2}$ ). Este intercambiador es muy 
adecuado de emplear para manejar fluidos a alta presión debido a los diámetros pequeños de las tuberías, y también bajo condiciones severas de ensuciamiento a causa de su facilidad de limpieza y mantenimiento. La principal desventaja que presentan es que son voluminosos y caros por unidad de área superficial de transferencia de calor (Kakaç et al., 2012).

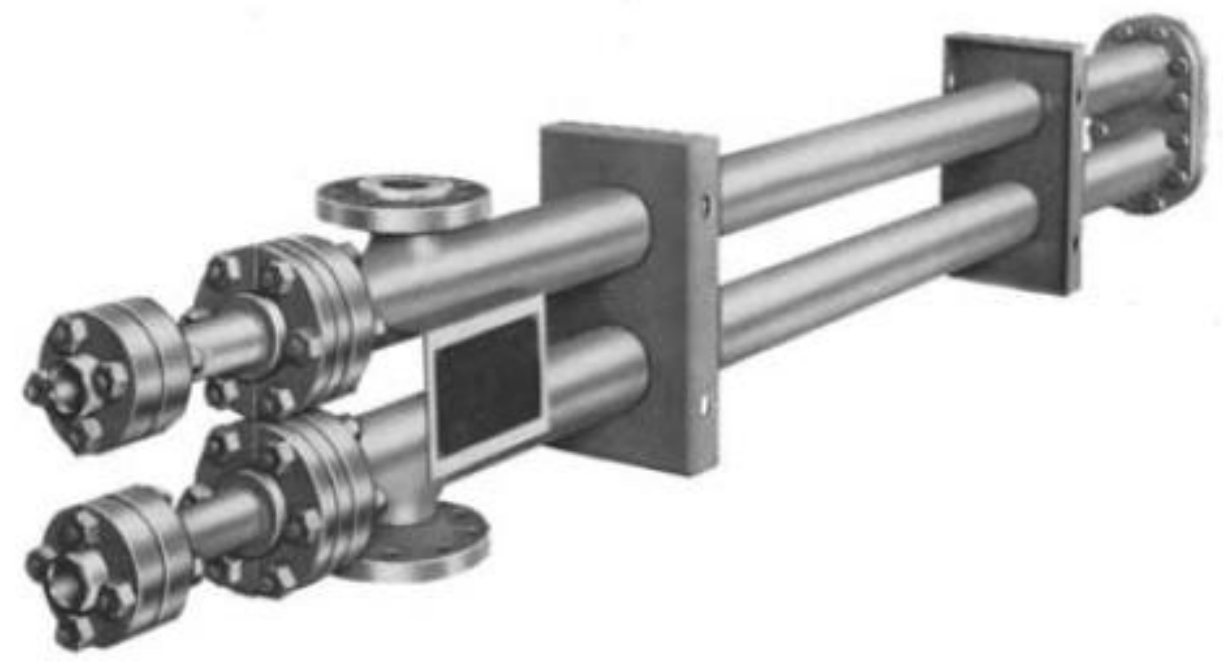

Figura 1. Intercambiador de calor de doble tubo típico (1 horquilla)

Fuente: Adaptado de (Kakaç et al., 2012)

Una forma de incrementar la capacidad de transferencia de calor de los tubos de un intercambiador de calor consiste en adjuntarle piezas de metal llamadas aletas, las cuales extienden la superficie de transferencia de calor. Esto hace posible tener una mayor superficie externa para una misma superficie interna. En aquellos casos donde el coeficiente de transferencia de calor controlante sea el externo, esto puede traer consigo un incremento significativo de la densidad de flujo de calor por unidad de área interna (Cao, 2010).

El intercambiador de calor de doble tubo con tubos aleteados constituye una alternativa muy atractiva para incrementar el rango de aplicación de estos equipos. Las aletas incrementan la superficie de transferencia de calor por unidad de longitud y reducen el tamaño y, por tanto, el número de horquillas requeridas para una determinada demanda de calor. Integralmente, las aletas longitudinales han demostrado ser las más eficientes para los intercambiadores de calor de doble tubo. Las aletas son más efectivas cuando el coeficiente pelicular es bajo. Cuando la carga de calor varía, pueden agregarse más horquillas para satisfacer la nueva demanda (Kakaç et al., 2012; Thulukkanam, 2013).

Son varios los autores que han evaluado la transferencia de calor en tubos aleteados de intercambiadores de calor de doble tubo. En este sentido (Syed et al., 2011) llevaron a cabo la optimización de un doble tubo aleteado con aletas trapezoidales utilizando un algoritmo genético y el método de Región de Confianza, con el objetivo de optimizar la configuración del ánulo aleteado determinado por el número de aletas, altura de la aleta, espesor de la aleta, y la relación de radios del tubo interno y externo, para el mejoramiento del coeficiente de transferencia de calor. También (Hameed \& Essa, 2015) desarrollaron investigaciones experimentales y numéricas para evaluar el rendimiento de un intercambiador de calor con aletas triangulares, empleando para ello el software COMSOL. Por otro lado (Kumar et al., 2015), compararon las características de transferencia de calor utilizando tres diferentes configuraciones de aletas (rectangular, triangular y parabólica cóncava) en un intercambiador de calor de doble tubo, aplicando varias condiciones de operación para obtener la mejor configuración posible. Además, (Omkar et al., 2014) estudiaron la transferencia de calor de un intercambiador de calor de doble tubo con aletas 
helicoidales instaladas en la superficie externa del tubo interior, mientras que se incrementó el nivel de turbulencia del proceso de intercambio de calor a partir de la rotación del tubo interior. Por último, (Iqbal et al., 2011) investigaron las configuraciones optimas de un ánulo aleteado con aletas parabólicas para obtener la convección máxima mediante el empleo de algoritmos genéticos y de Región de Confianza. En este trabajo se consideró el flujo estacionario, laminar, incompresible y totalmente desarrollado sujeto a condiciones de flujo de calor constantes.

Esta temática ha sido tratada igualmente de una forma u otra por diversos investigadores (Mir et al., 2004; Mon \& Gross, 2004; Rao \& Levy, 2008; Zhang et al., 2012; Ramu et al., 2017; Dalkılıç et al., 2020).

En el presente trabajo se realiza el diseño térmico de un intercambiador de calor de doble tubo con tubos aleteados longitudinales, con el fin de conocer parámetros de importancia tales como el número de horquillas, la caída de presión y la potencia de bombeo requerida para ambos fluidos. También se efectúan dos estudios de sensibilidad para evaluar cómo influye el incremento de tanto el caudal de alimentación como la temperatura de entrada del metanol sobre cuatro parámetros de diseño del intercambiador de calor de doble tubo, los cuales son: 1) Caída de presión del agua de enfriamiento; 2) Caída de presión del metanol; 3) Potencia de bombeo del agua de enfriamiento y 4) Potencia de bombeo del metanol.

\section{MATERIALES Y MÉTODOS}

\subsection{Definición del problema}

Se desea diseñar un intercambiador de calor de doble tubo con tubos aleteados para enfriar $5000 \mathrm{~kg} / \mathrm{h}$ $(1,39 \mathrm{~kg} / \mathrm{s})$ de una corriente de metanol desde $70^{\circ} \mathrm{C}$ hasta $30^{\circ} \mathrm{C}$ utilizando agua de enfriamiento a $5{ }^{\circ} \mathrm{C}$. Se desea que la temperatura de salida del agua no supere $\operatorname{los} 20^{\circ} \mathrm{C}$. Se disponen de tubos de $4 \mathrm{~m}$ de longitud, con un diámetro nominal de 2 pulg, mientras que el diámetro nominal del ánulo es de 3 pulg. Las aletas tienen una altura de $0,0127 \mathrm{~m}$, un espesor de $0,0009 \mathrm{~m}$, se dispone de un número de aletas por tubo igual a 30, y existe solo un tubo por el interior del ánulo (Fig. 2). El material de construcción del tubo interior es de acero al carbono, y los fluidos circulan a través del intercambiador bajo flujo a contracorriente. La caída de presión máxima permisible para el metanol y el agua de enfriamiento no deberán exceder los 100 000 y $10000 \mathrm{~Pa}$ respectivamente, y se considera una eficiencia isoentrópica de ambas bombas igual a 80 $\%$.
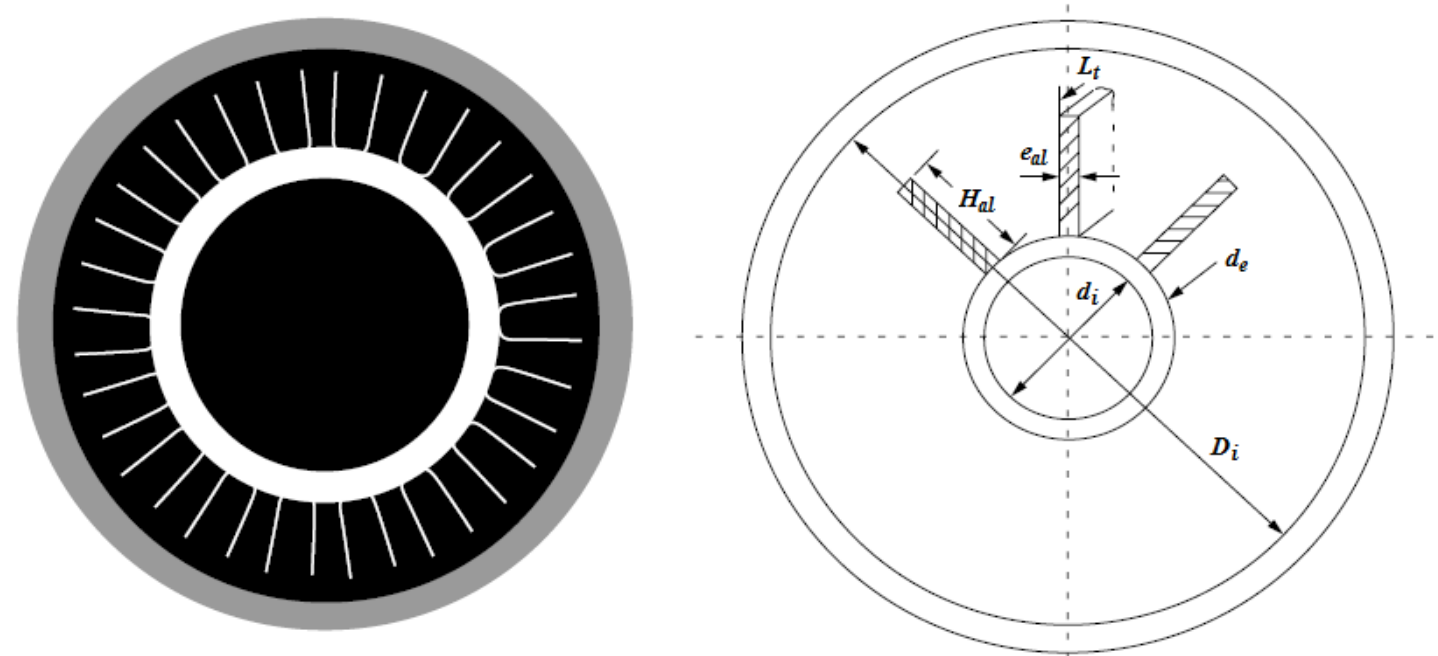

Figura 2. Sección transversal de un tubo interior con aletas longitudinales. Fuente: Adaptado de (Kakaç et al., 2012). 


\subsection{Metodología de cálculo a emplear}

Para realizar el diseño del intercambiador de doble tubo se emplearon correlaciones y ecuaciones publicadas fundamentalmente en (Kakaç et al., 2012), en donde se determina primeramente la cantidad de horquillas necesarias, para luego calcular las caídas de presión y por último la potencia de bombeo de ambos fluidos. Seguidamente se describen las etapas o pasos a seguir para efectuar el diseño del equipo.

\subsubsection{Numero de horquillas}

Paso 1. Definición de los datos iniciales requeridos para las corrientes involucradas:

Tabla 1. Datos iniciales requeridos para ambos fluidos involucrados en el sistema.

\begin{tabular}{lccc}
\hline Parámetro & Fluido frío & Fluido caliente & Unidades \\
\hline Caudal másico & $\mathrm{m}_{\mathrm{f}}$ & $\mathrm{m}_{\mathrm{c}}$ & $\mathrm{kg} / \mathrm{s}$ \\
Temperatura de entrada & $\mathrm{t}_{1}$ & $\mathrm{~T}_{1}$ & ${ }^{\circ} \mathrm{C}$ \\
Temperatura de salida & $\mathrm{t}_{2}$ & $\mathrm{~T}_{2}$ & ${ }^{\circ} \mathrm{C}$ \\
Caída de presión máxima permisible & $\Delta \mathrm{P}_{\mathrm{f}(\mathrm{p})}$ & $\Delta \mathrm{P}_{\mathrm{c}(\mathrm{p})}$ & ${ }_{\mathrm{Pa}}$ \\
Factor de ensuciamiento & $\mathrm{R}_{\mathrm{f}}$ & $\mathrm{R}_{\mathrm{c}}$ & $\mathrm{m}^{2} . \mathrm{K} / \mathrm{W}$ \\
\hline Fuente: Elaboración propia & & &
\end{tabular}

Fuente: Elaboración propia

Paso 2. Definición de los datos iniciales para el tubo interior, el ánulo y las aletas:

Tabla 2. Datos iniciales requeridos para el tubo interior, el ánulo y las aletas.

\begin{tabular}{lcc}
\hline Parámetro & Símbolo & Unidades \\
\hline Diámetro nominal del tubo interior & $\mathrm{d}_{\mathrm{n}}$ & $\mathrm{pulg}$ \\
Diámetro nominal del ánulo & $\mathrm{D}_{\mathrm{n}}$ & $\mathrm{pulg}$ \\
Altura de la aleta & $\mathrm{H}_{\mathrm{al}}$ & $\mathrm{m}$ \\
Espesor de la aleta & $\mathrm{e}_{\mathrm{al}}$ & $\mathrm{m}$ \\
Número de aletas por tubo & $\mathrm{N}_{\mathrm{al}}$ & - \\
Numero de tubos & $\mathrm{N}_{\mathrm{t}}$ & - \\
Longitud del tubo & $\mathrm{L}_{\mathrm{t}}$ & $\mathrm{m}$ \\
Conductividad térmica material del tubo interior & $\mathrm{k}_{\mathrm{t}}$ & $\mathrm{W} / \mathrm{m} . \mathrm{K}$ \\
\hline
\end{tabular}

Paso 3. Definición de los diámetros interiores y exteriores de ambas tuberías, según los diámetros nominales establecidos en el Paso 2.

Definir diámetro interior $\left(\mathrm{d}_{\mathrm{i}}\right)$ y exterior $\left(\mathrm{d}_{\mathrm{e}}\right)$ de la tubería interior, asi como el diámetro interior $\left(\mathrm{D}_{\mathrm{i}}\right)$ de la tubería exterior o ánulo.

Paso 3. Determinación de la temperatura media de ambas corrientes:

- Metanol:

$$
\bar{T}=\frac{T_{1}+T_{2}}{2}
$$

- Agua de enfriamiento: 


$$
\bar{t}=\frac{t_{1}+t_{2}}{2}
$$

Paso 4. Búsqueda de las propiedades físicas de cada fluido a la temperatura media determinada en el Paso 3.

Tabla 3. Propiedades físicas requeridas de cada fluido a la temperatura media calculada en el Paso 3.

\begin{tabular}{lccc}
\hline Propiedad & Fluido frío & Fluido caliente & Unidades \\
\hline Densidad & $\rho_{\mathrm{f}}$ & $\rho_{\mathrm{c}}$ & $\mathrm{kg} / \mathrm{m}^{3}$ \\
Calor específico & $\mathrm{Cp}_{\mathrm{f}}$ & $\mathrm{C} \mathrm{p}_{\mathrm{c}}$ & $\mathrm{J} / \mathrm{kg} . \mathrm{K}$ \\
Viscosidad & $\mu_{\mathrm{f}}$ & $\mu_{\mathrm{c}}$ & $\mathrm{Pa.s}$ \\
Conductividad térmica & $\mathrm{k}_{\mathrm{f}}$ & $\mathrm{k}_{\mathrm{c}}$ & $\mathrm{W} / \mathrm{m} . \mathrm{K}$ \\
\hline
\end{tabular}

Fuente: Elaboración propia

Paso 5. Cálculo de la carga de calor o calor intercambiado (Q):

La carga de calor o calor intercambiado se determina según los datos que se dispongan. De esta manera, si se tienen todos los datos de la corriente caliente, la carga de calor se determina según la siguiente ecuación:

$$
Q=m_{c} \cdot C p_{c} \cdot\left(T_{1}-T_{2}\right)
$$

Por otro lado, si se disponen de los datos de la corriente fría, entonces Q será:

$$
Q=m_{f} \cdot C p_{f} \cdot\left(t_{2}-t_{1}\right)
$$

Paso 6. Determinación del área neta transversal del ánulo con tubos aleteados longitudinales $\left(\mathrm{A}_{\mathrm{nt}}\right)$ :

$$
A_{n t}=\frac{\pi}{4} \cdot\left(D_{i}^{2}-d_{e}^{2} \cdot N_{t}\right)-\left(e_{a l} \cdot H_{a l} \cdot N_{a l} \cdot N_{t}\right)
$$

Paso 7. Determinación del perímetro húmedo total del ánulo con tubos interiores aleteados de forma longitudinal $\left(\mathrm{P}_{\mathrm{h}}\right)$ :

$$
P_{h}=\pi\left(D_{i}+d_{e} \cdot N_{t}\right)+2 \cdot H_{a l} \cdot N_{a l} \cdot N_{t}
$$

Paso 8. Cálculo del diámetro hidráulico $\left(\mathrm{D}_{\mathrm{h}}\right)$ :

$$
D_{h}=\frac{4 \cdot A_{n t}}{P_{h}}
$$

Paso 9. Cálculo del perímetro húmedo para la transferencia de calor $\left(\mathrm{P}_{\mathrm{hc}}\right)$ :

$$
P_{h c}=\pi \cdot d_{e} \cdot N_{t}+2 \cdot N_{a l} \cdot H_{a l} \cdot N_{t}
$$


Paso 10. Cálculo del diámetro equivalente para la transferencia de calor $\left(\mathrm{D}_{\mathrm{eq}}\right)$ :

$$
D_{e q}=\frac{4 \cdot A_{n t}}{P_{h c}}
$$

Paso 11. Cálculo del área de flujo del tubo interior $\left(a_{t}\right)$ :

$$
a_{t}=\frac{\pi \cdot d_{i}^{2}}{4}
$$

Paso 12. Ubicación de los fluidos en el intercambiador de calor

Paso 13. Determinación de la velocidad del fluido que circula por el tubo interior $\left(\mathrm{v}_{\mathrm{t}}\right)$ :

$$
v_{t}=\frac{m}{\rho \cdot a_{t}}
$$

Paso 14. Cálculo del número de Reynolds del fluido que circula por el tubo interior $\left(\operatorname{Re}_{t}\right)$ :

$$
\operatorname{Re}_{t}=\frac{\rho \cdot v_{t} \cdot d_{i}}{\mu}
$$

Paso 15. Determinación del número de Prandtl del fluido que circula por el tubo interior $\left(\operatorname{Pr}_{t}\right)$ :

$$
\operatorname{Pr}_{t}=\frac{C p \cdot \mu}{k}
$$

Paso 16. Determinación del número de Nusselt del fluido que circula por el tubo interior $\left(\mathrm{Nu}_{\mathrm{t}}\right)$ :

- Flujo laminar $\left(\operatorname{Re}_{t} \leq 2\right.$ 300):

Paso 16.1. Determinación de la temperatura de la pared del tubo:

$$
T_{p}=\frac{\bar{T}+\bar{t}}{2}
$$

Paso 16.2. Búsqueda de la viscosidad del fluido que circula por el tubo interior a la temperatura de la pared el tubo calculada en el paso 16.1.

Buscar $\mu_{\mathrm{p}}$ a $\mathrm{T}_{\mathrm{p}}$.

Paso 16.3. Cálculo del número de Nusselt del fluido que circula por el tubo interior bajo régimen laminar: 


$$
N u_{t}=1,86 \cdot\left(\operatorname{Re}_{t} \cdot \operatorname{Pr}_{t} \cdot \frac{d_{i}}{L_{t}}\right)^{1 / 3} \cdot\left(\frac{\mu}{\mu_{p}}\right)^{0,14}
$$

Válida para:

$0,48<\mathrm{Pr}_{\mathrm{t}}<16700$

- Flujo de transición $\left(2300<\mathrm{Re}_{\mathrm{t}}<10000\right)$ :

16.4. Determinación del factor de fricción de Fanning $\left(f_{t}\right)$ :

Válida para:

$$
f_{t}=\left(1,58 \cdot \ln \operatorname{Re}_{t}-3,28\right)^{-2}
$$

$2300<\operatorname{Re}_{\mathrm{t}}<5 \times 10^{6}$

$0,5<\mathrm{Pr}_{\mathrm{t}}<2000$

16.5. Cálculo del número de Nusselt para el fluido que circula por el tubo interior bajo régimen de transición:

$$
N u_{t}=\frac{\left(\frac{f_{t}}{2}\right) \cdot\left(\mathrm{Re}_{t}-1000\right) \cdot \operatorname{Pr}_{t}}{1+12,7 \cdot\left(\frac{f_{t}}{2}\right)^{0,5} \cdot\left(\operatorname{Pr}_{t}^{2 / 3}-1\right)}
$$

Válida para:

$2300<\mathrm{Re}_{\mathrm{t}}<10^{4}$

- Flujo turbulento $\left(\operatorname{Re}_{t} \geq 10000\right)$ :

Paso 16.6. Determinación del factor de fricción de Fanning $\left(f_{t}\right)$ :

Válida para:

$$
f_{t}=\left(1,58 \cdot \ln \operatorname{Re}_{t}-3,28\right)^{-2}
$$

$2300<\mathrm{Re}_{\mathrm{t}}<5 \times 10^{6}$

$0,5<\mathrm{Pr}_{\mathrm{t}}<2000$

16.7. Cálculo del número de Nusselt para el fluido que circula por el tubo interior bajo régimen turbulento:

$$
N u_{t}=\frac{\left(\frac{f_{t}}{2}\right) \cdot \operatorname{Re}_{t} \cdot \operatorname{Pr}_{t}}{1,07+12,7 \cdot\left(\frac{f_{t}}{2}\right)^{0,5} \cdot\left(\operatorname{Pr}_{t}^{2 / 3}-1\right)}
$$

Válida para:

$0,5<\operatorname{Pr}_{\mathrm{t}}<2000$ 
$10^{4}<\operatorname{Re}_{\mathrm{t}}<5 \times 10^{6}$

Paso 17. Determinación del coeficiente pelicular de transferencia de calor del fluido que circula por el tubo interior $\left(\mathrm{h}_{\mathrm{t}}\right)$ :

$$
h_{t}=\frac{N u_{t} \cdot k}{d_{i}}
$$

Paso 18. Determinación de la velocidad del fluido que circula por el ánulo $\left(\mathrm{v}_{\mathrm{a}}\right)$ :

$$
v_{a}=\frac{m}{\rho \cdot A_{n t}}
$$

Paso 19. Determinación del número de Reynolds del fluido que circula por el ánulo $\left(\operatorname{Re}_{\mathrm{a}}\right)$ :

$$
\operatorname{Re}_{a}=\frac{\rho \cdot v_{a} \cdot D_{h}}{\mu}
$$

Paso 20. Determinación del número de Prandtl del fluido que circula por el ánulo $\left(\operatorname{Pr}_{\mathrm{a}}\right)$ :

$$
\operatorname{Pr}_{a}=\frac{C p \cdot \mu}{k}
$$

Paso 21. Determinación del número de Nusselt del fluido que circula por el ánulo $\left(\mathrm{Nu}_{\mathrm{a}}\right)$ :

- Flujo laminar $\left(\operatorname{Re}_{\mathrm{a}} \leq \mathbf{2} 300\right)$

Paso 21.1. Búsqueda de la viscosidad del fluido que circula por el ánulo a la temperatura de la pared el tubo calculada en el paso 16.1.

Buscar $\mu_{\mathrm{p}}$ a $\mathrm{T}_{\mathrm{p}}$.

Paso 21.2. Cálculo del número de Nusselt del fluido que circula por el ánulo bajo régimen laminar:

$$
N u_{t}=1,86 \cdot\left(\operatorname{Re}_{a} \cdot \operatorname{Pr}_{a} \cdot \frac{D_{h}}{L_{t}}\right)^{1 / 3} \cdot\left(\frac{\mu}{\mu_{p}}\right)^{0,14}
$$

Válida para:

$0,48<\operatorname{Pr}_{\mathrm{a}}<16700$

- Flujo de transición $\left(2300<\operatorname{Re}_{\mathrm{a}}<10000\right)$ :

21.3. Determinación del factor de fricción de Fanning $\left(f_{a}\right)$ : 


$$
f_{a}=\left(1,58 \cdot \ln \operatorname{Re}_{a}-3,28\right)^{-2}
$$

Válida para:

$2300<\mathrm{Re}_{\mathrm{a}}<5 \times 10^{6}$

$0,5<\operatorname{Pr}_{a}<2000$

21.4. Cálculo del número de Nusselt para el fluido que circula por el ánulo bajo régimen de transición:

$$
N u_{a}=\frac{\left(\frac{f_{a}}{2}\right) \cdot\left(\operatorname{Re}_{a}-1000\right) \cdot \operatorname{Pr}_{a}}{1+12,7 \cdot\left(\frac{f_{a}}{2}\right)^{0,5} \cdot\left(\operatorname{Pr}_{a}^{2 / 3}-1\right)}
$$

Válida para:

$2300<\mathrm{Re}_{\mathrm{a}}<10^{4}$

- Flujo turbulento $\left(\operatorname{Re}_{\mathrm{a}} \geq 10000\right)$ :

Paso 21.5. Determinación del factor de fricción de Fanning $\left(f_{a}\right)$ :

$$
f_{a}=\left(1,58 \cdot \ln \operatorname{Re}_{a}-3,28\right)^{-2}
$$

Válida para:

$2300<\operatorname{Re}_{\mathrm{a}}<5 \times 10^{6}$

$0,5<\operatorname{Pr}_{\mathrm{a}}<2000$

Paso 21.6. Cálculo del número de Nusselt para el fluido que circula por el ánulo bajo régimen turbulento:

$$
N u_{a}=\frac{\left(\frac{f_{a}}{2}\right) \cdot \operatorname{Re}_{a} \cdot \operatorname{Pr}_{a}}{1,07+12,7 \cdot\left(\frac{f_{a}}{2}\right)^{0,5} \cdot\left(\operatorname{Pr}_{a}^{2 / 3}-1\right)}
$$

Válida para:

$0,5<\operatorname{Pr}_{\mathrm{a}}<2000$

$10^{4}<\mathrm{Re}_{\mathrm{a}}<5 \times 10^{6}$

Paso 22. Determinación del coeficiente pelicular de transferencia de calor del fluido que circula por el ánulo $\left(\mathrm{h}_{\mathrm{a}}\right)$ :

$$
h_{a}=\frac{N u_{a} \cdot k}{D_{e q}}
$$

Paso 23. Cálculo del área de transferencia de calor aleteada $\left(A_{a l}\right)$ : 


$$
A_{a l}=2 \cdot N_{t} \cdot N_{a l} \cdot L_{t} \cdot\left(2 \cdot H_{a l}+e_{a l}\right)
$$

Paso 24. Cálculo del área de transferencia de calor no aleteada $\left(A_{n}\right)$ :

$$
A_{n}=2 \cdot N_{t} \cdot\left(\pi \cdot d_{e} \cdot L_{t}-N_{a l} \cdot L_{t} \cdot e_{a l}\right)
$$

Paso 25. Determinación del área total de una horquilla $\left(A_{h}\right)$ :

$$
A_{h}=A_{n}+A_{a l}
$$

Paso 26. Determinación del parámetro m:

$$
m=\sqrt{\frac{2 \cdot h_{a}}{e_{a l} \cdot k_{t}}}
$$

Paso 27. Determinación de la eficiencia de la aleta $\left(\eta_{\mathrm{al}}\right)$ :

$$
\eta_{a l}=\frac{\tanh \left(m \cdot H_{a l}\right)}{m \cdot H_{a l}}
$$

Paso 28. Determinación de la eficiencia global superficial $\left(\eta_{\mathrm{s}}\right)$ :

$$
\eta_{s}=\left[1-\left(1-\eta_{a l}\right) \cdot \frac{A_{a l}}{A_{h}}\right]
$$

Paso 29. Determinación del área interna $\left(\mathrm{A}_{\mathrm{i}}\right)$ :

$$
A_{i}=2 \cdot \pi \cdot d_{i} \cdot L_{t}
$$

Paso 30. Cálculo del coeficiente global de transferencia de calor sucio $\left(\mathrm{U}_{\mathrm{S}}\right)$ :

$$
U_{S}=\frac{1}{\frac{A_{h}}{A_{i}} \cdot \frac{1}{h_{t}}+\frac{A_{h}}{A_{i}} \cdot R+\frac{A_{h} \cdot \ln \left(\frac{d_{e}}{d_{i}}\right)}{2 \cdot \pi \cdot k_{t} \cdot 2 \cdot L_{t}}+\frac{R}{\eta_{s}}+\frac{1}{\eta_{s} \cdot h_{a}}}
$$

Paso 31. Cálculo del coeficiente global de transferencia de calor limpio $\left(\mathrm{U}_{\mathrm{L}}\right)$ : 


$$
U_{L}=\frac{1}{\frac{A_{h}}{A_{i}} \cdot \frac{1}{h_{t}}+\frac{A_{h} \cdot \ln \left(\frac{d_{e}}{d_{i}}\right)}{2 \cdot \pi \cdot k_{t} \cdot 2 \cdot L_{t}}+\frac{1}{\eta_{s} \cdot h_{a}}}
$$

Paso 32. Cálculo del factor de limpieza $\left(\mathrm{F}_{\mathrm{L}}\right)$ :

$$
F_{L}=\frac{U_{S}}{U_{L}}
$$

Paso 33. Cálculo de la Media Logarítmica de la Diferencia de Temperatura (MLDT):

Para flujo a contracorriente:

$$
M L D T=\frac{\left(T_{1}-t_{2}\right)-\left(T_{2}-t_{1}\right)}{\ln \frac{\left(T_{1}-t_{2}\right)}{\left(T_{2}-t_{1}\right)}}
$$

Paso 34. Determinación del área total de transferencia de calor sin considerar ensuciamiento $\left(A_{L}\right)$ :

$$
A_{L}=\frac{Q}{U_{L} \cdot M L D T}
$$

Paso 35. Determinación del área total de transferencia de calor bajo condiciones de ensuciamiento $\left(\mathrm{A}_{\mathrm{S}}\right)$ :

$$
A_{S}=\frac{Q}{U_{S} \cdot M L D T}
$$

Paso 36. Cálculo del número de horquillas necesarias $\left(\mathrm{N}_{\mathrm{h}}\right)$ :

$$
N_{h}=\frac{A_{S}}{A_{h}}
$$

\subsubsection{Caída de presión}

Paso 37. Cálculo del factor de fricción del fluido que circula por el tubo interior:

- Flujo laminar $(\operatorname{Re} \leq 2300)$ :

$$
f_{\operatorname{lam}(t)}=\frac{16}{\mathrm{Re}_{t}}
$$


- $\quad$ Flujo turbulento $(\operatorname{Re}>2300)$ :

$$
f_{\text {turb(t) }}=\left(1,58 \cdot \ln \operatorname{Re}_{t}-3,28\right)^{-2}
$$

Paso 38. Corrección del factor de fricción si se cumple que el fluido que circula por el tubo interior fluye bajo régimen laminar $\left(f_{\operatorname{lam}(t)}^{\prime}\right)$ :

- Calentamiento del fluido que circula por el tubo interior:

$$
f_{\text {lam }(t)}^{\prime}=f_{\text {lam }(t)} \cdot\left(\frac{\mu}{\mu_{p}}\right)^{-0,58}
$$

- Enfriamiento del fluido que circula por el tubo interior:

$$
f_{\text {lam }(t)}^{\prime}=f_{\text {lam }(t)} \cdot\left(\frac{\mu}{\mu_{p}}\right)^{-0,50}
$$

Paso 39. Cálculo de la caída de presión del fluido que circula por el tubo interior:

- Flujo laminar $(\operatorname{Re} \leq 2300)$ :

$$
\Delta p_{t}=4 \cdot f^{\prime}{ }_{\operatorname{lam}(t)} \cdot \frac{2 \cdot L_{t}}{d_{i}} \cdot \rho \cdot \frac{v_{t}^{2}}{2} \cdot N_{h}
$$

- Flujo turbulento $(\operatorname{Re}>2300)$ :

$$
\Delta p_{t}=4 \cdot f_{t u r b(t)} \cdot \frac{2 \cdot L_{t}}{d_{i}} \cdot \rho \cdot \frac{v_{t}^{2}}{2} \cdot N_{h}
$$

Paso 40. Factor de fricción del fluido que circula por el ánulo:

- Flujo laminar $(\operatorname{Re} \leq 2300)$ :

$$
f_{\text {lam }(a)}=\frac{16}{\operatorname{Re}_{a}}
$$

- $\quad$ Flujo turbulento $(\operatorname{Re}>2300)$ : 


$$
f_{\text {turb }(a)}=\left(1,58 \cdot \ln \operatorname{Re}_{a}-3,28\right)^{-2}
$$

Paso 41. Corrección del factor de fricción si se cumple que el fluido que circula por el ánulo fluye bajo régimen laminar $\left[f_{\operatorname{lam}(a)}\right]$ :

- Calentamiento del fluido que circula por el ánulo:

$$
f_{\text {lam }(a)}^{\prime}=f_{\text {lam }(a)} \cdot\left(\frac{\mu}{\mu_{p}}\right)^{-0,58}
$$

- Enfriamiento del fluido que circula por el ánulo:

$$
f_{\text {lam }(a)}^{\prime}=f_{\text {lam }(a)} \cdot\left(\frac{\mu}{\mu_{p}}\right)^{-0,50}
$$

Paso 42. Caída de presión del fluido que circula por el ánulo $\left(\Delta \mathrm{p}_{\mathrm{a}}\right)$ :

- Flujo laminar $(\operatorname{Re} \leq 2300)$ :

$$
\Delta p_{a}=4 \cdot f^{\prime}{ }_{\operatorname{lam}(a)} \cdot \frac{2 \cdot L_{t}}{D_{h}} \cdot \rho \cdot \frac{v_{a}^{2}}{2} \cdot N_{h}
$$

- $\quad$ Flujo turbulento $(\operatorname{Re}>2300)$ :

$$
\Delta p_{a}=4 \cdot f_{\operatorname{turb}(a)} \cdot \frac{2 \cdot L_{t}}{D_{h}} \cdot \rho \cdot \frac{v_{a}^{2}}{2} \cdot N_{h}
$$

\subsubsection{Potencia de bombeo}

Paso 43. Potencia de bombeo del fluido que circula por el tubo interior $\left(\mathrm{P}_{\mathrm{bt}}\right)$ :

$$
P_{b t}=\frac{\Delta p_{t} \cdot m}{\eta_{b} \cdot \rho}
$$

Paso 44. Potencia de bombeo del fluido que circula por el ánulo $\left(\mathrm{P}_{\mathrm{ba}}\right)$ :

$$
P_{b a}=\frac{\Delta p_{a} \cdot m}{\eta_{b} \cdot \rho}
$$

Donde $\eta_{\mathrm{b}}$ es 0,80 según datos iniciales. 


\subsection{Estudios de sensibilidad}

Se efectuaron dos estudios de sensibilidad para determinar la influencia que presenta el incremento de dos parámetros iniciales de la corriente de metanol sobre cuatro variables de diseño del intercambiador de calor de doble tubo. Los parámetros iniciales a modificar son: 1) el caudal másico de alimentación del metanol y 2) la temperatura de entrada del metanol, mientras que las variables de salida a evaluar son: 1) la caída de presión del agua de enfriamiento; 2) la caída de presión del metanol; 3) la potencia de bombeo del agua de enfriamiento, y 4) la potencia de bombeo del metanol. En el caso del caudal de alimentación del metanol, este se varió en el rango de $5000-7000 \mathrm{~kg} / \mathrm{h}$, mientras que en el caso de la temperatura de entrada del metanol, se modificó en el intervalo de $60-80{ }^{\circ} \mathrm{C}$.

\section{RESULTADOS Y DISCUSIÓN}

Seguidamente se muestran los resultados alcanzados al realizar los cálculos de los diferentes parámetros incluidos en la metodología de diseño del intercambiador de doble tubo con tubos aleteados.

\subsection{Número de horquillas}

Paso 1. Definición de los datos iniciales requeridos para cada corriente de fluido:

Tabla 4. Valores de los datos iniciales requeridos para cada fluido.

\begin{tabular}{lccc}
\hline Parámetro & Agua & Metanol & Unidades \\
\hline Caudal másico & - & 1,39 & $\mathrm{~kg} / \mathrm{s}$ \\
Temperatura de entrada & 5 & 70 & ${ }^{\circ} \mathrm{C}$ \\
Temperatura de salida & 20 & 30 & ${ }^{\circ} \mathrm{C}$ \\
Caída de presión máxima permisible & 10000 & 100000 & $\mathrm{~Pa}$ \\
Factor de ensuciamiento & 0,000176 & 0,000352 & $\mathrm{~m}^{2} . \mathrm{K} / \mathrm{W}$ \\
\hline Funte: Elaboción propia
\end{tabular}

Paso 2. Definición de los datos iniciales para el tubo interior, el ánulo y las aletas:

Tabla 5. Valores de los datos iniciales requeridos para el tubo interior, el ánulo y las aletas.

\begin{tabular}{lccc}
\hline Parámetro & Símbolo & Valor & Unidades \\
\hline Diámetro nominal del tubo interior & $\mathrm{d}_{\mathrm{n}}$ & 2 & $\mathrm{pulg}$ \\
Diámetro nominal del ánulo & $\mathrm{D}_{\mathrm{n}}$ & 3 & $\mathrm{pulg}$ \\
Altura de la aleta & $\mathrm{H}_{\mathrm{al}}$ & 0,0127 & $\mathrm{~m}$ \\
Espesor de la aleta & $\mathrm{e}_{\mathrm{al}}$ & 0,0009 & $\mathrm{~m}$ \\
Número de aletas por tubo & $\mathrm{N}_{\mathrm{al}}$ & 30 & - \\
Numero de tubos & $\mathrm{N}_{\mathrm{t}}$ & 1 & 1 \\
Longitud del tubo & $\mathrm{L}_{\mathrm{t}}$ & 4 & $\mathrm{~m}$ \\
Conductividad térmica material del tubo interior & $\mathrm{k}_{\mathrm{t}}$ & 52 & $\mathrm{~W} / \mathrm{m} . \mathrm{K}$ \\
\hline Fuente: Elaboración propia & & &
\end{tabular}

Paso 3. Definición de los diámetros interiores y exteriores de ambas tuberías, según los diámetros nominales establecidos en el Paso 2.

Según (Perry \& Green, 2008) tanto el tubo interior como el ánulo presentarán los siguientes diámetros:

- Diámetro interior del tubo interior $\left(\mathrm{d}_{\mathrm{i}}\right): 0,05250 \mathrm{~m}$. 
- Diámetro exterior del tubo interior $\left(\mathrm{d}_{\mathrm{e}}\right): 0,06033 \mathrm{~m}$.

- Diámetro interior ánulo $\left(\mathrm{D}_{\mathrm{i}}\right): 0,07793 \mathrm{~m}$.

Paso 3. Temperatura media de ambas corrientes:

- Metanol:

$$
\bar{T}=\frac{T_{1}+T_{2}}{2}=\frac{70+30}{2}=50^{\circ} \mathrm{C}
$$

- Agua de enfriamiento:

$$
\bar{t}=\frac{t_{1}+t_{2}}{2}=\frac{5+20}{2}=12,5^{\circ} \mathrm{C}
$$

Paso 4. Búsqueda de las propiedades físicas de cada fluido a la temperatura media determinada en el Paso 3.

Según (Perry \& Green, 2008), tanto el agua de enfriamiento como el metanol presentan los siguientes valores de las propiedades físicas a la temperatura media determinada en el Paso 3.

Tabla 6. Valores de las propiedades físicas de ambos fluidos a la temperatura media determinada en el Paso 3.

\begin{tabular}{lccc}
\hline Propiedad & Agua & Metanol & Unidades \\
\hline Densidad & 1002,92 & 764,90 & $\mathrm{~kg} / \mathrm{m}^{3}$ \\
Calor específico & 4200,44 & 2705,40 & $\mathrm{~J} / \mathrm{kg} . \mathrm{K}$ \\
Viscosidad & 0,001223 & 0,000399 & $\mathrm{Pa.s}$ \\
Conductividad térmica & 0,5877 & 0,1929 & $\mathrm{~W} / \mathrm{m} . \mathrm{K}$ \\
\hline
\end{tabular}

Fuente: Elaboración propia

Paso 5. Carga de calor o calor intercambiado (Q):

Como se disponen de todos los datos para la corriente caliente, entonces Q se determinará mediante la ecuación (3). Así:

$$
Q=m_{c} \cdot C p_{c} \cdot\left(T_{1}-T_{2}\right)=150420,4 W
$$

Luego, el caudal necesario de agua de enfriamiento será:

$$
m_{f}=\frac{Q}{C p_{f} \cdot\left(t_{2}-t_{1}\right)}=\frac{150420,4}{4200,44 \cdot(20-5)}=2,39 \mathrm{~kg} / \mathrm{s}
$$

La Tabla 1 muestra los resultados de los parámetros determinados en los pasos 6-11.

Tabla 7. Resultados de los parámetros incluidos en los pasos $6-11$.

\begin{tabular}{clccc}
\hline Paso & Parámetro & Símbolo & Valor & Unidades \\
\hline 6 & $\begin{array}{l}\text { Área neta transversal del ánulo con tubos aleteados } \\
\text { longitudinales }\end{array}$ & $\mathrm{A}_{\mathrm{nt}}$ & 0,001567 & $\mathrm{~m}^{2}$ \\
7 & $\begin{array}{l}\text { Perímetro húmedo total del ánulo con tubos interiores } \\
\mathrm{P}_{\mathrm{h}}\end{array}$ & 1,196 & $\mathrm{~m}$
\end{tabular}




\section{aleteados de forma longitudinal}

8 Diámetro hidráulico

9 Perímetro húmedo para la transferencia de calor

10 Diámetro equivalente para la transferencia de calor

11 Área de flujo del tubo interior

Fuente: Elaboración propia

Paso 12. Ubicación de los fluidos en el intercambiador de calor:

Siguiendo recomendaciones y sugerencias publicadas en (Sinnott, 2005), se ubica el agua de enfriamiento por el tubo interior y el metanol por el ánulo.

La Tabla 2 expone los resultados de los parámetros determinados en los pasos 13 - 15.

Tabla 8. Resultados de los parámetros determinados en los pasos 13 - 15 .

\begin{tabular}{clccc}
\hline Paso & Parámetro & Símbolo & Valor & Unidades \\
\hline 13 & Velocidad del agua de enfriamiento & $\mathrm{V}_{\mathrm{t}}$ & 1,10 & $\mathrm{~m} / \mathrm{s}$ \\
14 & Número de Reynolds del agua de enfriamiento & $\mathrm{Re}_{\mathrm{t}}$ & 47357,83 & - \\
15 & Número de Prandtl del agua de enfriamiento & $\operatorname{Pr}_{\mathrm{t}}$ & 8,74 & - \\
\hline Fuente: Elaboración propia & &
\end{tabular}

Paso 16. Número de Nusselt del agua de enfriamiento $\left(\mathrm{Nu}_{\mathrm{t}}\right)$ :

Como el agua de enfriamiento fluye bajo régimen de flujo turbulento pues se cumple que $\operatorname{Re}_{\mathrm{t}}>10000$, entonces se hará uso directamente de los pasos 16.6 y 16.7. De esta manera:

Paso 16.6. Factor de fricción de Fanning del agua de enfriamiento bajo régimen turbulento $\left(f_{t}\right)$ :

$f_{t}=\left(1,58 \cdot \ln \operatorname{Re}_{t}-3,28\right)^{-2}=0,0053$

Paso 16.7. Número de Nusselt del agua de enfriamiento bajo régimen turbulento $\left(\mathrm{Nu}_{\mathrm{t}}\right)$ :

$N u_{t}=\frac{\left(\frac{f_{t}}{2}\right) \cdot \operatorname{Re}_{t} \cdot \operatorname{Pr}_{t}}{1,07+12,7 \cdot\left(\frac{f_{t}}{2}\right)^{0,5} \cdot\left(\operatorname{Pr}_{t}^{2 / 3}-1\right)}=343,65$

La Tabla 3 describe los parámetros determinados en los pasos 17 - 20.

Tabla 9. Resultados de los parámetros determinados en los pasos 17 - 20.

\begin{tabular}{clccc}
\hline Paso & Parámetro & Símbolo & Valor & Unidades \\
\hline 17 & Coeficiente pelicular de transferencia de calor del & $\mathrm{h}_{\mathrm{t}}$ & 3846,92 & $\mathrm{~W} / \mathrm{m}^{2} . \mathrm{K}$ \\
& agua de enfriamiento & & \\
18 & Velocidad del metanol & $\mathrm{v}_{\mathrm{a}}$ & 1,16 & $\mathrm{~m} / \mathrm{s}$ \\
19 & Número de Reynolds del metanol & $\mathrm{Re}_{\mathrm{a}}$ & 11652,55 & - \\
20 & Número de Prandtl del metanol & $\mathrm{Pr}_{\mathrm{a}}$ & 5,60 & - \\
\hline Fuente: Elaboración propia & & &
\end{tabular}

Paso 21. Número de Nusselt del metanol $\left(\mathrm{Nu}_{\mathrm{a}}\right)$ : 
Según el resultado obtenido en el Paso 19, el metanol fluye bajo flujo turbulento por el ánulo, ya que se cumple que $\mathrm{Re}_{\mathrm{a}}>10$ 000. Luego, se hará uso de los pasos 21.5 y 21.6 para determinar el número de Nusselt.

Paso 21.5. Factor de fricción de Fanning del metanol bajo régimen turbulento $\left(f_{a}\right)$ :

$$
f_{a}=\left(1,58 \cdot \ln \operatorname{Re}_{a}-3,28\right)^{-2}=0,0075
$$

Paso 21.6. Número de Nusselt del agua de enfriamiento bajo régimen turbulento $\left(\mathrm{Nu}_{\mathrm{t}}\right)$ :

$$
N u_{a}=\frac{\left(\frac{f_{a}}{2}\right) \cdot \operatorname{Re}_{a} \cdot \operatorname{Pr}_{a}}{1,07+12,7 \cdot\left(\frac{f_{a}}{2}\right)^{0,5} \cdot\left(\operatorname{Pr}_{a}^{2 / 3}-1\right)}=89,15
$$

La Tabla 4 presenta los resultados de los parámetros determinados en los pasos 22 - 35 .

Tabla 10. Resultados de los parámetros determinados en los pasos $22-35$.

\begin{tabular}{clccc}
\hline Paso & Parámetro & Símbolo & Valor & Unidades \\
\hline 22 & Coeficiente pelicular de transferencia de calor del & $\mathrm{h}_{\mathrm{a}}$ & 2609,56 & $\mathrm{~W} / \mathrm{m}^{2} \cdot \mathrm{K}$ \\
& metanol & $\mathrm{A}_{\mathrm{al}}$ & 6,312 & \\
23 & Área de transferencia de calor aleteada & $\mathrm{A}_{\mathrm{n}}$ & 1,299 & $\mathrm{~m}^{2}$ \\
24 & Área de transferencia de calor no aleteada & $\mathrm{A}_{\mathrm{h}}$ & 7,611 & $\mathrm{~m}^{2}$ \\
25 & Área total de una horquilla & $\mathrm{m}$ & 333,95 & - \\
26 & Parámetro m & $\eta_{\mathrm{al}}$ & 0,236 & - \\
27 & Eficiencia de la aleta & $\eta_{\mathrm{s}}$ & 0,366 & - \\
28 & Efíciencia global superficial & $\mathrm{A}_{\mathrm{i}}$ & 1,319 & $\mathrm{~m}^{2}$ \\
29 & Área interna & $\mathrm{U}_{\mathrm{S}}$ & 202,86 & $\mathrm{~W} / \mathrm{m}^{2} \cdot \mathrm{K}$ \\
30 & Coeficiente global de transferencia de calor sucio & $\mathrm{U}_{\mathrm{L}}$ & 338,76 & $\mathrm{~W} / \mathrm{m}^{2} \cdot \mathrm{K}$ \\
31 & Coeficiente global de transferencia de calor limpio & $\mathrm{F}_{\mathrm{L}}$ & 0,60 & - \\
32 & Factor de limpieza & $\mathrm{MLDT}$ & 36,07 & ${ }^{\circ} \mathrm{C}$ \\
33 & Media Logarítmica de la Diferencia de Temperatura & 12,31 & $\mathrm{~m}^{2}$ \\
34 & Área total de transferencia de calor sin considerar & $\mathrm{A}_{\mathrm{L}}$ & & \\
& ensuciamiento & & & $\mathrm{m}^{2}$ \\
35 & Área total de transferencia de calor bajo condiciones & $\mathrm{As}_{\mathrm{S}}$ & 20,56 & \\
& de ensuciamiento & & &
\end{tabular}

Paso 36. Número de horquillas $\left(\mathrm{N}_{\mathrm{h}}\right)$ :

$N_{h}=\frac{A_{S}}{A_{h}}=\frac{20,56}{7,611}=2,7 \approx 3$

\subsection{Caída de presión}

Paso 37. Factor de fricción del agua de enfriamiento $\left(f_{\operatorname{turb}(t)}\right)$ : 
Como el agua de enfriamiento fluye bajo régimen de flujo turbulento, entonces se hará uso de la ecuación (16) para determinar el factor de fricción de este fluido. Entonces:

$$
f_{\text {turb }(t)}=\left(1,58 \cdot \ln \operatorname{Re}_{t}-3,28\right)^{-2}=0,0053
$$

Paso 39. Caída de presión del agua de enfriamiento:

Se hará uso de la ecuación (46) para flujo turbulento ( $\operatorname{Re}>2300)$ :

$$
\Delta p_{t}=4 \cdot f_{\text {turb }(t)} \cdot \frac{2 \cdot L_{t}}{d_{i}} \cdot \rho_{f} \cdot \frac{v_{t}^{2}}{2} \cdot N_{h}=5880,39 P a
$$

Paso 40. Factor de fricción del metanol $\left(f_{\operatorname{turb}(a)}\right)$ :

Debido a que el metanol fluye también bajo régimen turbulento, entonces el factor de fricción de este fluido se determinará mediante la ecuación (24). Luego:

$$
f_{\text {turb(a) }}=\left(1,58 \cdot \ln \operatorname{Re}_{a}-3,28\right)^{-2}=0,0075
$$

Paso 42 Caída de presión del metanol:

Se hará uso de la ecuación (51) para régimen turbulento:

$$
\Delta p_{a}=4 \cdot f_{t u r b(a)} \cdot \frac{2 \cdot L_{t}}{D_{h}} \cdot \rho_{c} \cdot \frac{v_{a}^{2}}{2} \cdot N_{h}=70711,91 P a
$$

\subsection{Potencia de bombeo}

43. Potencia de bombeo del agua de enfriamiento:

$$
P_{b t}=\frac{\Delta p_{t} \cdot m_{f}}{\eta_{b} \cdot \rho_{f}}=17,52 \mathrm{~W}
$$

44. Potencia de bombeo del metanol:

$$
P_{b a}=\frac{\Delta p_{a} \cdot m_{c}}{\eta_{b} \cdot \rho_{c}}=160,62 \mathrm{~W}
$$

Según los resultados obtenidos, ambos fluidos se mueven bajo régimen turbulento debido a que tanto el número de Reynolds del agua de enfriamiento $(47$ 357,83) como del metanol $(11652,55)$ se encuentran por encima de 10000 . El coeficiente pelicular de transferencia de calor del agua de enfriamiento (3 846,92 $\left.\mathrm{W} / \mathrm{m}^{2} . \mathrm{K}\right)$ es 1,47 veces superior que el coeficiente pelicular de transferencia de calor del metanol (2 $\left.609,56 \mathrm{~W} / \mathrm{m}^{2} . \mathrm{K}\right)$. 
El intercambiador de calor de doble tubo posee una eficiencia de la aleta de 0,236 , un coeficiente global de transferencia de calor considerando ensuciamiento de $202,86 \mathrm{~W} / \mathrm{m}^{2} . \mathrm{K}$, un factor de limpieza de 0,60 , un área de transferencia de calor bajo condiciones de ensuciamiento de $20,56 \mathrm{~m}^{2}$, y un número total de horquillas igual a tres. Tanto la caída de presión del agua de enfriamiento (5 880,39 Pa) como la del metanol $(70711,91 \mathrm{~Pa})$ se encuentran por debajo de los límites máximos permisibles establecidos por el proceso, los cuales son $100000 \mathrm{~Pa}$ y $10000 \mathrm{~Pa}$ para el metanol y el agua de enfriamiento, respectivamente. Se observa que la caída de presión del metanol es 12,03 veces superior que la caída de presión del agua de enfriamiento.

Por último, se necesita una potencia de bombeo para impulsar el agua de enfriamiento y el metanol de $17,52 \mathrm{~W}$ y 160,62 W, respectivamente. Vale destacar que la potencia de bombeo del metanol es 9,17 veces superior que la potencia de bombeo del agua de enfriamiento, lo cual se debe, fundamentalmente, a la mayor caída de presión obtenida para el metanol.

\subsection{Estudio de sensibilidad No. 1.}

La Figura 3 muestra los resultados obtenidos al efectuar el primer estudio de sensibilidad, consistente en determinar la influencia que presenta un incremento del caudal de alimentación del metanol $\left(\mathrm{m}_{\mathrm{c}}\right)$ sobre cuatro parámetros de diseño del intercambiador.

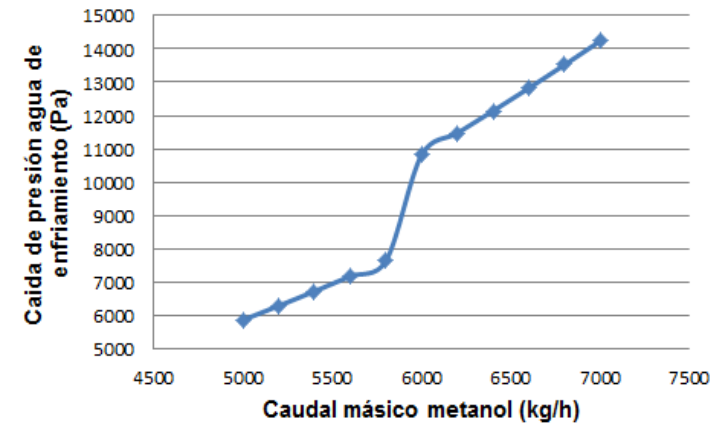

(a)

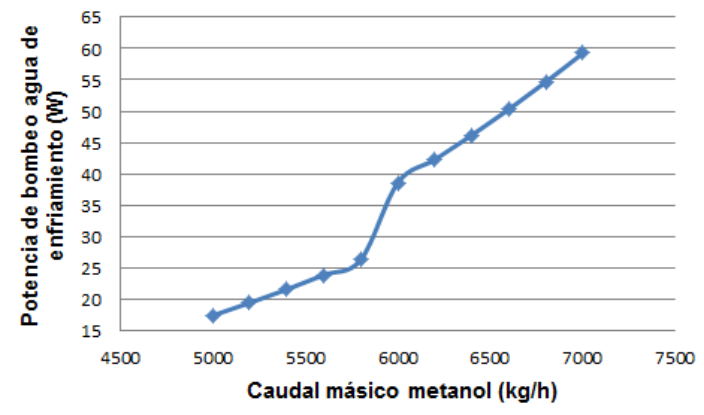

(c)

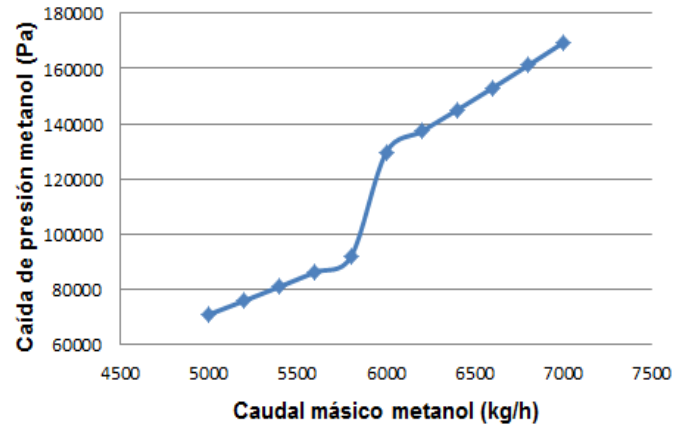

(b)

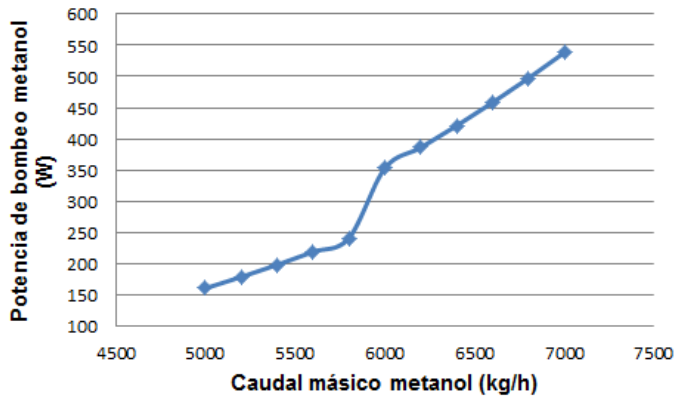

(d)

Figura 3. Influencia del incremento del caudal de alimentación del metanol sobre cuatro parámetros de diseño del intercambiador de doble tubo. (a) Caída de presión del agua de enfriamiento. (b) Caída de presión del metanol. (c) Potencia de bombeo del agua de enfriamiento. (d) Potencia de bombeo del metanol.

Fuente: Elaboración propia

De acuerdo con los resultados mostrados en la Figura 3, todos los parámetros evaluados se incrementan con un aumento del caudal de alimentación del metanol. Es de resaltar que a partir de los $5860 \mathrm{~kg} / \mathrm{h}$ tanto 
la caída de presión del agua de enfriamiento como del metanol superan el límite máximo establecido por el proceso ambas corrientes. Se recomienda de esta manera que el equipo opere con un caudal de alimentación de metanol preferiblemente por debajo de los $5800 \mathrm{~kg} / \mathrm{h}$ para que la caída de presión de ambas corrientes se mantenga dentro de los límites fijados y la potencia de bombeo no se incremente hasta alcanzar valores prohibidamente elevados.

\subsection{Estudio de sensibilidad No. 2.}

La Figura 4 expone los resultados obtenidos al efectuar el estudio de sensibilidad No. 2, consistente en determinar la influencia que presenta un aumento de la temperatura de entrada del metanol $\left(\mathrm{T}_{1}\right)$ sobre cuatro parámetros de diseño del intercambiador.

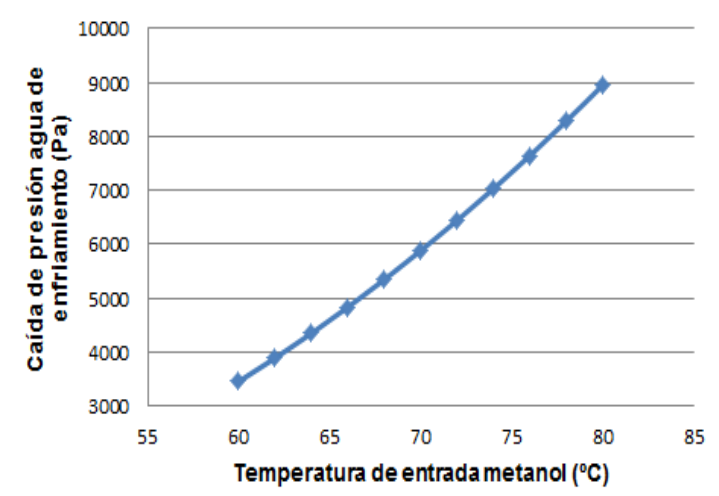

(a)

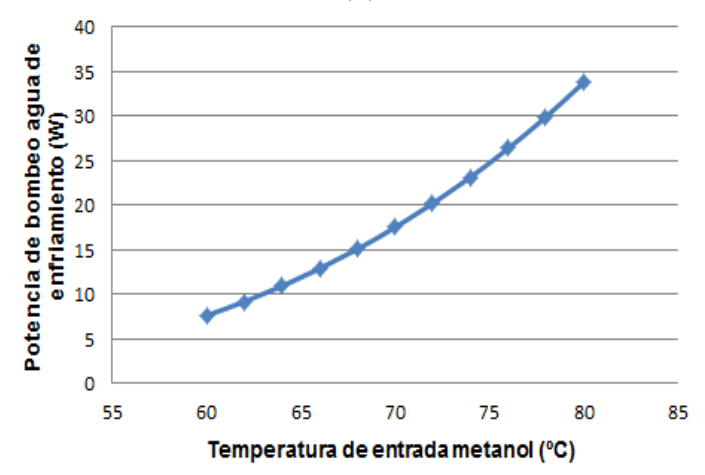

(c)

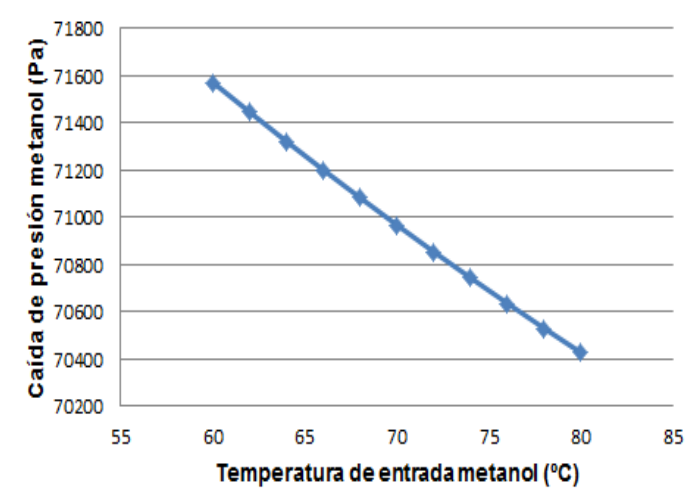

(b)

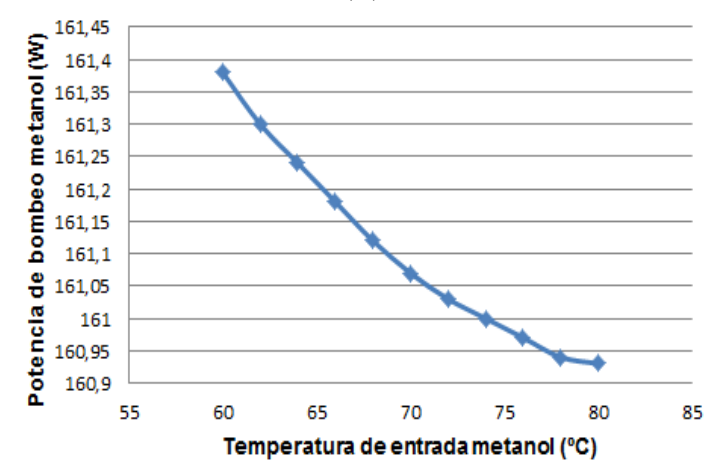

(d)

Figura 4. Influencia del incremento de la temperatura de entrada del metanol sobre cuatro parámetros de diseño del intercambiador de doble tubo. a) Caída de presión del agua de enfriamiento. (b) Caída de presión del metanol. (c) Potencia de bombeo del agua de enfriamiento. (d) Potencia de bombeo del metanol.

Fuente: Elaboración propia

Considerando los resultados mostrados en la Figura 4, se puede decir que tanto la caída de presión como la potencia de bombeo del agua de enfriamiento aumentan con un incremento de la temperatura de entrada del metanol $\left(\mathrm{T}_{1}\right)$, mientras que tanto la caída de presión como la potencia de bombeo del metanol disminuyen con el incremento de $\mathrm{T}_{1}$. Se puede observar además que $\mathrm{T}_{1}$ puede alcanzar un valor de $80^{\circ} \mathrm{C}$ y la caída de presión del agua de enfriamiento aún se mantiene por debajo del valor máximo establecido para esta corriente $(10000 \mathrm{~Pa})$. Se resume entonces que $\mathrm{T}_{1}$ puede incrementarse hasta alcanzar un valor de $80{ }^{\circ} \mathrm{C}$ sin que esto afecte negativamente la caída de presión del agua de enfriamiento. La potencia de bombeo del agua de enfriamiento, por su parte, se incrementa hasta alcanzar un máximo de 33,76 W para 
un valor de $\mathrm{T}_{1}$ de $80{ }^{\circ} \mathrm{C}$. El incremento de $\mathrm{T}_{1}$ desde $60^{\circ} \mathrm{C}$ hasta $80^{\circ} \mathrm{C}$ incrementa la potencia de bombeo del agua de enfriamiento en $26,17 \mathrm{~W}$ (o 4,45 veces).

\section{CONCLUSIONES}

1. Ambos fluidos fluyen bajo régimen turbulento ya que tanto el número de Reynolds del agua de enfriamiento $(47357,83)$ como del metanol $(11652,55)$ se encuentran por encima de 10000.

2. El coeficiente pelicular de transferencia de calor del agua de enfriamiento ( $3846,92 \mathrm{~W} / \mathrm{m}^{2} . \mathrm{K}$ ) es 1,47 veces superior que el coeficiente pelicular de transferencia de calor del metanol $\left(2609,56 \mathrm{~W} / \mathrm{m}^{2} . \mathrm{K}\right)$.

3. El intercambiador de calor de doble tubo posee una eficiencia de la aleta de 0,236 , un coeficiente global de transferencia de calor considerando ensuciamiento de $202,86 \mathrm{~W} / \mathrm{m}^{2} . \mathrm{K}$, un factor de limpieza de 0,60 y un área de transferencia de calor bajo condiciones de ensuciamiento de 20,56 $\mathrm{m}^{2}$.

4. El intercambiador de calor de doble tubo diseñado tendrá tres horquillas.

5. Tanto la caída de presión calculada del agua de enfriamiento ( $5880,39 \mathrm{~Pa}$ ) como la del metanol (70 $711,91 \mathrm{~Pa}$ ) se encuentran por debajo de los límites máximos fijados por el proceso.

6. La potencia de bombeo necesaria para impulsar tanto el agua de enfriamiento como el metanol alcanzó valores de 17,52 W y 160,62 W, respectivamente.

7. Los cuatro parámetros evaluados en el estudio de sensibilidad No. 1 se incrementan con un aumento del caudal de alimentación del metanol, mientras que se aconseja que el equipo opere con un caudal de alimentación de metanol preferiblemente por debajo de los $5800 \mathrm{~kg} / \mathrm{h}$ para que la caída de presión de ambas corrientes se mantenga dentro de los límites fijados.

8. Tanto la caída de presión como la potencia de bombeo del agua de enfriamiento aumentan con un incremento de la temperatura de entrada del metanol $\left(\mathrm{T}_{1}\right)$, mientras que tanto la caída de presión como la potencia de bombeo del metanol disminuyen con el incremento de $\mathrm{T}_{1}$.

9. La temperatura de entrada del metanol puede incrementarse hasta $80{ }^{\circ} \mathrm{C}$ sin que esto incremente las caídas de presión de ambos fluidos por encima de los límites máximos permisibles.

\section{REFERENCIAS}

Cao, E. (2010). Heat transfer in process engineering. New York, USA: McGraw-Hill.

Dalkılıç, A. S., Mercan, H., Özçelik, G., and Wongwises, S. (2020). Optimization of the finned doublepipe heat exchanger using nanofluids as working fluids. Journal of Thermal Analysis and Calorimetry, 120. doi: 10.1007/s10973-020-09290-x.

Hameed, V. M., and Essa, B. M. (2015). Experimental and numerical investigation to evaluate the performance of triangular finned tube heat exchanger. International Journal of Energy and Environment, 6 (6), 553-566. 
Iqbal, Z., Syed, K. S., and Ishaq, M. (2011). Optimal convective heat transfer in double pipe with parabolic fins. International Journal of Heat and Mass Transfer, 54, 5415-5426. doi: 10.1016/j.ijheatmasstransfer.2011.08.001.

Kakaç, S., Liu, H., and Pramuanjaroenkij, A. (2012). Heat Exchangers. Selection, Rating and Thermal Design. Boca Raton, USA: CRC Press.

Kumar, S., Karanth, K. V., and Murthy, K. (2015). Numerical study of heat transfer in a finned double pipe heat exchanger. World Journal of Modelling and Simulation, 11 (1), 43-54.

Mir, N. A., Syed, K. S., and Iqbal, M. (2004). Numerical Solution of Fluid Flow and Heat Transfer in the Finned Double Pipe. Journal of Research (Science), 15 (3), 253-262.

Mon, M. S., and Gross, U. (2004). Numerical study of fin-spacing effects in annular-finned tube heat exchangers. International Journal of Heat and Mass Transfer, 47 (8-9), 1953-1964. doi: 10.1016/j.ijheatmasstransfer.2003.09.034.

Omkar, M. S., Pravin, A. M., Sajid, A. H. G., and Pradeep, A. P. (2014). Experimental investigation of double-pipe heat exchanger with helical fins on the inner rotating tube. International Journal of Research in Engineering and Technology, 3 (7), 98-102.

Perry, R. H., and Green, D. W. (2008). Perry's Chemical Engineers' Handbook. New York, USA: McGraw-Hill.

Ramu, S., Achamyeleh, T., Srinivasan, R., and KrishnaKumar, K. (2017). Analysis of Heat Generation in Double Pipe Heat Exchanger: An Experimental Evaluation and Comparison of the Heat Transfer rate in the Surface Contact of the Elliptical Fin. International Research Journal of Engineering and Technology, 4 (6), 1279-1284.

Rao, G. A., and Levy, Y. (2008). A Semi Empirical Methodology for Performance Estimation of a Double Pipe Finned Heat Exchanger. 9th Biennial ASME Conference on Engineering Systems Design and Analysis (ESDA08), Haifa, Israel.

Sinnott, R. K. (2005). Chemical Engineering Design (5th ed. Vol. 6). Oxford, UK: Elsevier ButterworthHeinemann.

Syed, K. S., Iqbal, Z., and Ishaq, M. (2011). Optimal configuration of finned annulus in a double pipe with fully developed laminar flow. Applied Thermal Engineering, 31, 1435-1446. doi: 10.1016/j.applthermaleng.2011.01.012.

Thulukkanam, K. (2013). Heat Exchanger Design Handbook (2nd ed.). Boca Raton, USA: CRC Press.

Zhang, L., Du, W., Wu, J., Li, Y., and Xing, Y. (2012). Fluid flow characteristics for shell side of doublepipe heat exchanger with helical fins and pin fins. Experimental Thermal and Fluid Science, 36, 30-43. doi: 10.1016/j.expthermflusci.2011.08.001.

Zohuri, B. (2017). Compact Heat Exchangers - Selection, Application, Design and Evaluation. Switzerland: Springer International Publishing.

\section{SEMBLANZA DE LOS AUTORES}




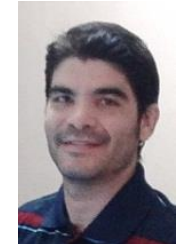

Amaury Pérez Sánchez: Obtuvo el grado de Ingeniero Químico en la Universidad de Camagüey, Cuba, en el año 2009, donde actualmente es profesor instructor e investigador auxiliar. En estos momentos se encuentra estudiando un Máster en Biotecnología en el centro de Ingeniería Genética y Biotecnología de Camagüey. Trabaja en líneas de investigación vinculadas fundamentalmente con el diseño de equipos de transferencia de calor y masa, simulación y optimización de procesos y operaciones de la industria química mediante el empleo de simuladores, y la evaluación técnicoeconómica de procesos y plantas biotecnológicas.

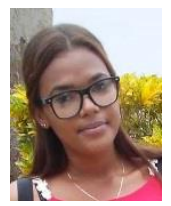

Greisy Ivety Valero Almanza: Obtuvo el grado de Ingeniero Quimico en la Universidad de Camagüey, Cuba en el año 2019. Se desempeña profesonalmente como Especialista de Ciencia, Tecnología y Medio Ambiente en el Departamento de Gestión Ambiental del Centro de Ingenieria Ambiental de Camagüey. Su área de investigación comprende la realización de estudios de riesgos mediombientales en empresas y fábricas quimicas, gestión medioambiental de residuales y desechos químicos, análisis fisico-quimico de aguas y aguas residuales, asi como tambien el diseño y evaluación de equipos de transferencia de calor y masa.

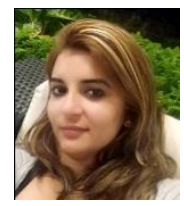

Elizabeth Ranero González. Obtuvo el grado de Ingeniero Químico en la Universidad de Camagüey, Cuba en el año 2016. Se desempeña profesionalmente como Profesor Instructor en la Universidad de Camagüey, Cuba. Su área de investigación abarca el diseño y evaluación de equipos y procesos de transferencia de calor y masa, evaluación termodinámica de procesos químicos, y la simulación de procesos y sistemas biotecnológicos.

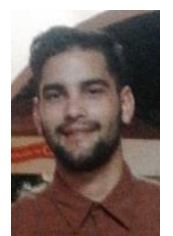

Eddy Javier Pérez Sánchez: Obtuvo el grado de Ingeniero Químico en la Universidad de Camagüey, Cuba en el año 2016. Se desempeña profesionalmente en la Empresa de Servicios Automotores S.A., de la ciudad de Ciego de Ávila, Cuba, en el Departamento de Dirección Comercial. Trabaja en líneas de investigación relacionadas con el diseño, evaluación y/o operación de equipos de transferencia de calor y masa, así como también la simulación de procesos petroquímicos y biotecnológicos. 